Copyright (C) 2014 IEEE. Personal use of this material is permitted. Permission from IEEE must be obtained for all other uses, in any current or future media, including reprinting/republishing this material for advertising or promotional purposes, creating new collective works, for resale or redistribution to servers or lists, or reuse of any copyrighted component of this work in other works. 


\section{Improving the Murchison Widefield Array Tile Model for Polarimetry}

\author{
Adrian Sutinjo, \\ Shantanu Padhi, \\ Randall Wayth, \\ Peter Hall \\ and Steven Tingay \\ Curtin University \\ 1 Turner Avenue \\ Bentley, Western Australia \\ Email: adrian.sutinjo@icrar.org
}

\author{
John O'Sullivan \\ Formerly \\ CSIRO Centre Astronomy \\ and Space Science (CASS) \\ Email: josulliv@bigpond.net.au
}

\author{
Emil Lenc \\ Sydney Institute for Astronomy \\ School of Physics \\ The University of Sydney \\ NSW, Australia \\ ARC Centre of Excellence \\ for All-sky Astrophysics (CAASTRO) \\ Sydney, Australia \\ Email: e.lenc@physics.usyd.edu.au
}

\begin{abstract}
This paper summarizes the progress to date in improving the Murchison Widefield Array's (MWA) "tile" model for polarimetry. A simple model that accounts for inter-element mutual coupling is presented which explains the false polarization leakage in Stokes Q ("false Q") seen when the array is scanned away from zenith in the principal planes. On these planes the model predicts current imbalance in the $X(E-W)$ and $Y(N$ S) dipoles and hence the false $Q$. More sophisticated models intended to calibrate actual observation data will be briefly discussed.
\end{abstract}

\section{INTRODUCTION}

The Murchison Widefield Array (MWA) [1], [2] is one of the precursors to the low frequency Square Kilometer Array (SKA-low) situated in the Murchison Radio-astronomy Observatory (MRO) in the mid-west of Western Australia. The array consists of 128 "tiles" each of which is a regularly spaced $4 \times 4$ bow-tie antennas as seen in Fig. 1. A longstanding issue in MWA polarimetry is that calibration gain values for an MWA pointing angle do not transfer to other pointing angles [3] which manifests most prominently as "false Q". As shown in Fig. 2, this is particularly severe at high frequencies (e.g., $>200 \mathrm{MHz}$ ) where $Q / I$ for an unpolarized source may be as high as a couple tens of percent. At lower frequencies around $100-140 \mathrm{MHz}$, this error is of the order of a few percent.

Here we present a simple model that explains the features of the false Q leakages. We point out that this model is "skeletal" in nature intended to highlight the primary mechanisms for the the appearance of false Q. A more sophisticated "calibrationgrade" model is under investigation.

\section{Simple Model}

We consider the array in transmission which is well known to be equal to the receive behavior using the principle of reciprocity. At every frequency, the voltage delays and port currents are related via an impedance matrix which in the case

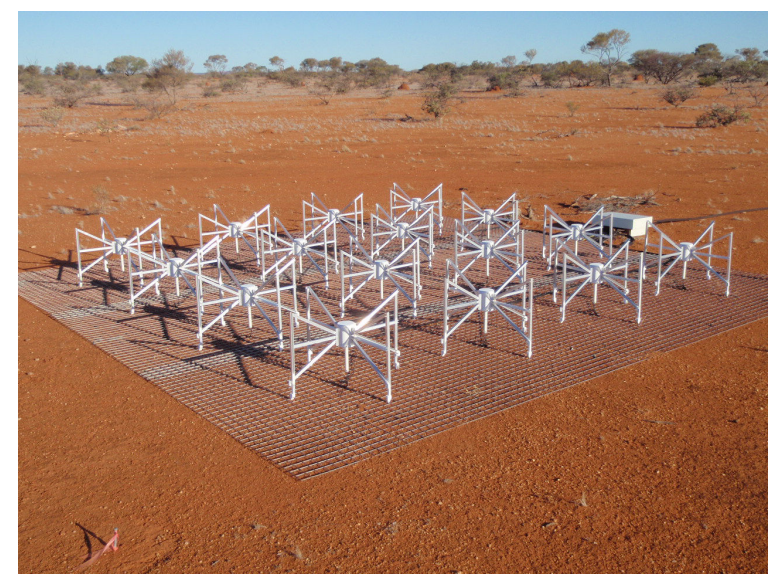

Fig. 1. An photo of an MWA tile. The bow-ties are regularly spaced at $1.1 \mathrm{~m}$ on a $5 \times 5 \mathrm{~m}$ wire grid and were designed to operate from $80-300 \mathrm{MHz}$. The bow-tie arms are oriented E-W and N-S.

of MWA is $32 \times 32$ [4] corresponding to 16 " $\mathrm{X}$ " and 16 " $\mathrm{Y}$ " dipoles.

$$
\mathbf{v}=\left(\mathbf{Z}+\mathbf{Z}_{L}\right) \mathbf{i}
$$

where $\mathbf{v}=\left[v_{1} \ldots v_{16}, v_{17} \ldots v_{32}\right]^{T}$ is the known "excitation" voltage vector, $\mathbf{i}=\left[i_{1} \ldots i_{16}, i_{17} \ldots i_{32}\right]^{T}$ is the unknown port current vector and $\mathbf{Z}$ describes the inter-port $\mathbf{Z}$ parameter coupling matrix [4] and $\mathbf{Z}_{L}$ is a diagonal matrix containing the LNA input impedances.

The unknown port currents are obtained simply by

$$
\mathbf{i}=\left(\mathbf{Z}+\mathbf{Z}_{L}\right)^{-1} \mathbf{v}
$$

The appearance of false Q is illustrated in Fig. 3 at $200 \mathrm{MHz}$ for MWA pointing to zenith and for $A z=0^{\circ}, E l=54^{\circ}$. While the current amplitudes are very similar when zenith pointing, the difference in the " $\mathrm{X}$ " and " $\mathrm{Y}$ " dipole current amplitudes for $E l=54^{\circ}$ is in the tens of percent. This imbalance is 


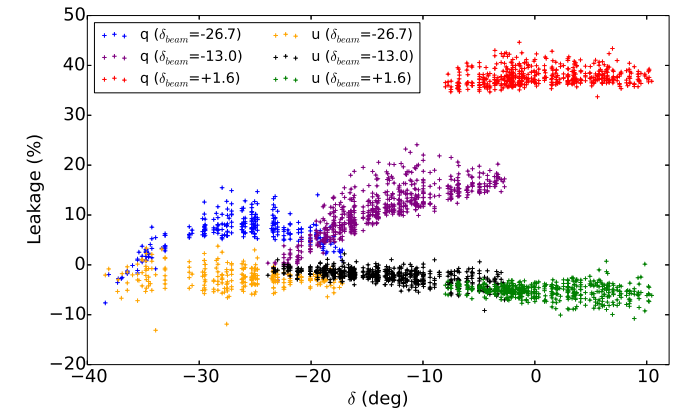

Fig. 2. Percentage polarization leakage $(\mathrm{Q} / \mathrm{I}$ and $\mathrm{U} / \mathrm{I})$ measured for bright radio sources at various declinations that drift through the field of view with three different beam-former settings for scan angles along the meridian. The 200-230 MHz data was calibrated with the source 3C444 (9.7 degrees north off zenith).

commensurate to false Q as existing MWA tile model neglects mutual coupling and therefore does not recognize this effect.
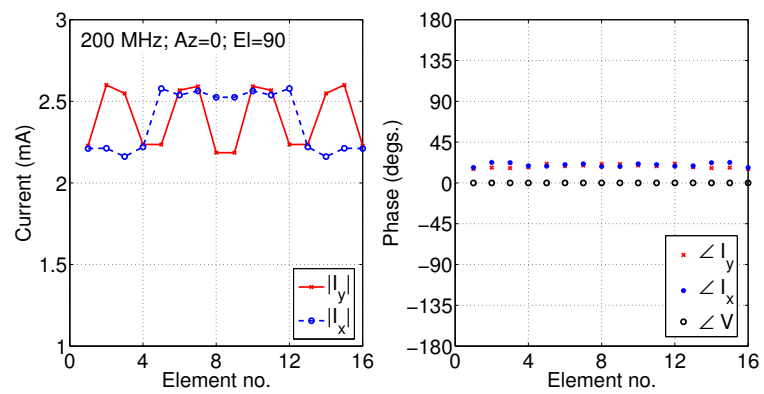

(a)
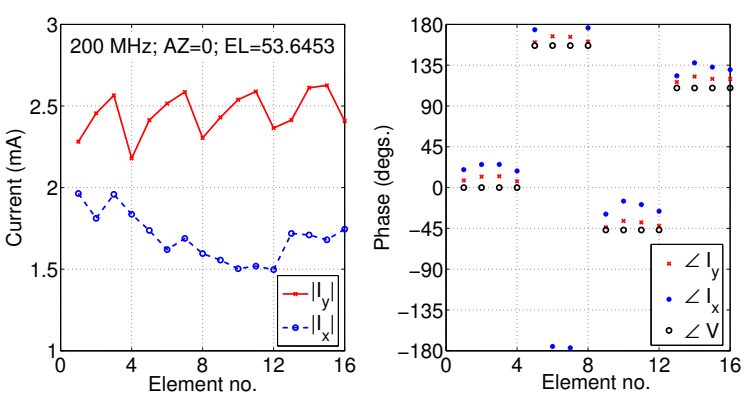

(b)

Fig. 3. Port current distribution for an MWA tile over an infinite perfectly conducting (PEC) ground plane at $200 \mathrm{MHz}$ for $A z=0^{\circ}$ for two tile pointings: (a) zenith and (b) $E l \approx 54^{\circ}$ both of which are MWA "sweetspots" (i.e., the intended pointing direction for a set of discrete MWA delay steps).

\section{JONES MATRIX MODEL}

The foregoing model is simplified and is intended to show only the essence of the problem. A more complete model is likely required for the purpose of accurately calibrating observation data. A key simplifying assumption there is that the element patterns are identical which is not necessarily the case for a "small" finite array such as an MWA [5]. More rigorous model is likely required for the purpose of calibrating observation data. Such a model may be developed by summing the "embedded element pattern" for the elements calculated by electromagnetic modelling for a unit current excitation of each port. This is under investigation.

\section{CONCLUSION}

The appearance of very high "false Q" in the principal planes can be attributed to current imbalance in the $\mathrm{X}$ and $\mathrm{Y}$ MWA bow-ties. A simple model based on impedance matrix has been shown to be sufficient to replicate the features of the error. We expect improve this model with more rigorous model in the near future.

\section{ACKNOWLEDGMENTS}

We acknowledge the Wajarri Yamatji people as the traditional owners of the Murchison Radio Astronomy Observatory site. The MRO is operated by CSIRO, whose assistance we acknowledge. The Centre for All-sky Astrophysics (CAASTRO) is an Australian Research Council Centre of Excellence, funded by grant CE110001020

\section{REFERENCES}

[1] C. Lonsdale, R. Cappallo, M. Morales, F. Briggs, L. Benkevitch, J. Bowman, J. Bunton, S. Burns, B. Corey, L. deSouza, S. Doeleman, M. Derome, A. Deshpande, M. Gopala, L. Greenhill, D. Herne, J. Hewitt, P. Kamini, J. Kasper, B. Kincaid, J. Kocz, E. Kowald, E. Kratzenberg, D. Kumar, M. Lynch, S. Madhavi, M. Matejek, D. Mitchell, E. Morgan, D. Oberoi, S. Ord, J. Pathikulangara, T. Prabu, A. Rogers, A. Roshi, J. Salah, R. Sault, N. Shankar, K. Srivani, J. Stevens, S. Tingay, A. Vaccarella, M. Waterson, R. Wayth, R. Webster, A. Whitney, A. Williams, and C. Williams, "The murchison widefield array: Design overview," Proceedings of the IEEE, vol. 97, no. 8, pp. $1497-1506$, aug. 2009.

[2] S. J. Tingay, R. Goeke, J. D. Bowman, D. Emrich, S. M. Ord, D. A. Mitchell, M. F. Morales, T. Booler, B. Crosse, R. B. Wayth, C. J. Lonsdale, S. Tremblay, D. Pallot, T. Colegate, A. Wicenec, N. Kudryavtseva, W. Arcus, D. Barnes, G. Bernardi, F. Briggs, S. Burns, J. D. Bunton, R. J. Cappallo, B. E. Corey, A. Deshpande, L. Desouza, B. M. Gaensler, L. J. Greenhill, P. J. Hall, B. J. Hazelton, D. Herne, J. N. Hewitt, M. Johnston-Hollitt, D. L. Kaplan, J. C. Kasper, B. B. Kincaid, R. Koenig, E. Kratzenberg, M. J. Lynch, B. Mckinley, S. R. Mcwhirter, E. Morgan, D. Oberoi, J. Pathikulangara, T. Prabu, R. A. Remillard, A. E. E. Rogers, A. Roshi, J. E. Salah, R. J. Sault, N. Udaya-Shankar, F. Schlagenhaufer, K. S. Srivani, J. Stevens, R. Subrahmanyan, M. Waterson, R. L. Webster, A. R. Whitney, A. Williams, C. L. Williams, and J. S. B. Wyithe, "The Murchison Widefield Array: The Square Kilometre Array Precursor at Low Radio Frequencies," Publications of the Astronomical Society of Australia, vol. 30, p. 7, Jan. 2013.

[3] S. M. Ord, D. A. Mitchell, R. B. Wayth, L. J. Greenhill, G. Bernardi, S. Gleadow, R. G. Edgar, M. A. Clark, G. Allen, W. Arcus, L. Benkevitch, J. D. Bowman, F. H. Briggs, J. D. Bunton, S. Burns, R. J. Cappallo, W. A. Coles, B. E. Corey, L. deSouza, S. S. Doeleman, M. Derome, A. Deshpande, D. Emrich, R. Goeke, M. R. Gopalakrishna, D. Herne, J. N. Hewitt, P. A. Kamini, D. L. Kaplan, J. C. Kasper, B. B. Kincaid, J. Kocz, E. Kowald, E. Kratzenberg, D. Kumar, C. J. Lonsdale, M. J. Lynch, S. R. McWhirter, S. Madhavi, M. Matejek, M. F. Morales, E. Morgan, D. Oberoi, J. Pathikulangara, T. Prabu, A. E. E. Rogers, A. Roshi, J. E. Salah, A. Schinkel, N. U. Shankar, K. S. Srivani, J. Stevens, S. J. Tingay, A. Vaccarella, M. Waterson, R. L. Webster, A. R. Whitney, A. Williams, and C. Williams, "Interferometric imaging with the 32 element murchison wide-field array," Publications of the Astronomical Society of the Pacific, vol. 122, no. 897, pp. pp. 1353-1366, 2010. [Online]. Available: http://www.jstor.org/stable/10.1086/657160

[4] W. L. Stutzman and G. A. Thiele, Antenna Theory and Design, 2nd ed. Wiley, 1998.

[5] D. Kelley and W. Stutzman, "Array antenna pattern modeling methods that include mutual coupling effects," Antennas and Propagation, IEEE Transactions on, vol. 41, no. 12, pp. 1625-1632, 1993. 